\title{
Dynamically regulated miRNA-mRNA network in lymph node metastasis of prostate cancer patients
}

\author{
M. Shkurnikov*, E. Knyazev, D. Mikhailenko, A. Zotikov, B. Alekseev \\ National Medical Research Radiology Center, Obninsk, Russia \\ *e-mail:mshkurnikov@imscs.msu.ru
}

Key words: prostate cancer, metastasis, microarrays

Motivation and Aim: The lymph node metastasis in prostate cancer is associated with poor prognosis of overall survival of patients. Recent evidences suggest that metastatic activity of tumors could be regulated by extracellular circulating microRNAs which are released by various cells of the body. In this study we have analyzed the microRNA profiles in blood plasma of prostate cancer patients as well as the expression of microRNA-targeted genes in prostate cancer tumors with and without metastases to lymph nodes.

Methods and Algorithms: Blood plasmas from overall 101 prostate cancer patients (89$\mathrm{pN}_{0} \mathrm{M}_{0}$ stage, and $12-\mathrm{pN}_{1} \mathrm{M}_{0}$ stage) have been collected in a period between 20152016, and profiled for extracellular microRNAs using GeneChip miRNA 4.0 Arrays (Affymetrix, USA). The microRNAs that showed at least 2 -fold ( $p$-value $<0.05$ ) change in expression between the analyzed groups of patients were selected for the subsequent analysis. Next, the list of mRNA genes targeted by at least two of the pre-selected circulating microRNAs simultaneously, was compiled. The expression data of these genes in tumors of prostate cancer patients with $(N=75)$ and without $(N=321)$ lymph node metastases were obtained from the TCGA database. All experiments have been conducted in accordance with the principles of the Declaration of Helsinki of World Medical Association.

Results: The analysis of microRNA profiles in blood plasmas of prostate cancer patients with lymph node metastases has revealed a significant decrease in the levels of nine circulating microRNAs including hsa-miR-92a-3p, hsa-miR-16-5p, hsa-miR-451a, hsamiR-93-5p, hsa-let-7c-5p, hsa-miR-320a, hsa-miR-17-5p, hsa-miR-106a-5p, and hsamiR-25-3p. We hypothesize that these microRNAs could be secreted by cells from tumor microenvironment, or other distant tissues (including the immune system components) and suppress tumor metastasis. Furthermore, elevated expression of six mRNA-coding genes (including BIRC5, CDC20, MELK, RRM2, UBE2C,TUBB3), which were among the potential targets for the pre-selected nine miRNAs, was observed in tumor tissues from patients with lymph node metastases. Finally, each of these mRNA genes was an independent predictor of cancer metastasis with AUC $>0.66$ and adjusted p-value was less than 0.05 .

Conclusion: Our data demonstrate the role of nine circulating microRNAs in suppressing the lymph nodes metastases during prostate cancer, presumably by inhibiting the expression of six target genes in tumor tissues. These findings could be applied for the development of new approaches for stage differentiation and treatment of prostate cancer. Acknowledgements: Supported by the MES of Russia (14.616.21.0092, RFMEFI61618X0092). 